\title{
Balanced Parameters for Genotype X Environment Interaction in Some Alfalfa Genotypes
}

\author{
Mofeeda Seiam ${ }^{1}$ and Sahar A. Farag ${ }^{2}$ \\ ${ }^{1}$ Field Crops Research Institute, ARC, Nubaria, Egypt. \\ ${ }^{2}$ Central Laboratory for Design \& Statistical Analysis Research, ARC, Giza, Egypt.
}

\begin{abstract}
Identification of alfalfa genotypes, with high yield potential and good forage quality along with the least seasonal fluctuation over a wide range of environments, is important for crop improvement programs. Trials were conducted to evaluate seven alfalfa (Medicago sativa, L) genotypes over three years at the Nubaria Agricultural Research Station Site. Field experiments were carried out in 2014, 2015 and 2016 seasons to evaluate fresh and dry forage yield, protein and fiber percentages and their stability employing parametric and non-parametric measures for the seven genotypes across environments. Results indicated that the local cultivar Ismailia-1 was superior to the introduced genotypes regarding its fresh and dry forage yield in addition to recording the least fiber percentage; however, MI reya produced higher protein percentage. When stability measures for the four traits was considered, Isamilia-1 was considered the most stable for fiber percentage based on four different measures tested. MI reya was most stable for protein percentage and more stable than Ismailia-1 for fresh and dry forage yield based on estimates from larger number of the tested stability measures, suggesting that MI reya is the most promising genotype among the introduced genotypes under study.
\end{abstract}

Key wards: Parametric and nonparametric stability, genetic stability, dry forage yield, Genotype $\mathrm{x}$ environment.

\section{INTRODUCTION}

Alfalfa (Medicago sativa L.) is a major forage crop in many countries around the world, where, it is cultivated over a wide range of climatic and edaphic conditions ranging from the semi-arid regions to the humid areas. It is recognized as high quality forage for all classes of livestock. In Egypt almost about 90 thousand feddans are grown to alfalfa annually, whereas, demand for the crop is on rise (CAPMAS 2019). Data on the crop showed that, about $18 \%$ increase in area devoted to alfalfa has been recorded from the growing season $2014 / 2015$ to $2016 / 2017$, however, the increase in production was only $7 \%$ suggesting the need for improvement in crop productivity. Because alfalfa is adapted to conditions of excess heat, drought and salinity, it is preferred than berseem clover in newly reclaimed areas of Nubaria, Ismailia and the 1.5 million feddan reclamation project launched by the Egyptian government in 2014. The need for new cultivars with higher productivity and better feed quality is necessary to cope with the increased demand on the crop.

The decision to select a variety is usually made on the basis of whether the variety's performance is stable and satisfactory in comparison to the performance of commercial ones. Consequently, to develop a variety with high yielding ability and consistency, high attention during selection should be given to the stability performance of the genotypes under different environments and their interaction (Allard and Bradshow 1964). Oushy et al. (1999) reported that alfalfa landraces from the New Valley were superior to the introduced genotypes and that the introduced genotypes could be used to reconstruct elite synthetic varieties. Furthermore, Bakheit (1988) reported that only two out of 18 introduced genotypes showed promising forage yields with good phenotypic and genotypic stability indices compared to local cultivars to be selected for ongoing breeding programs for alfalfa improvement.

To evaluate genotypes, Finally and Wilkinson (1963) proposed the average yield of all genotypes grown at particular site in a particular season as a measure of that environment, and used the regression coefficient (b) of the varietal means in its environment as an indicator for the phenotypic stability and adaptation. Nascimento et al. (2013), evaluated a methodology of adaptability and phenotypic stability of 92 alfalfa genotypes, where they obtained 20 cuttings along the growing seasons of Nov. 2004 to June 2006 and considered each cut as one environment. Different measures for stability are currently available including; non-parametric measures as the one described by Nassar and Huehn (1987), but also parametric measures for phenotypic stability including Wricke (1962), Eberhart and Russell, (1966), Shukla (1972) Pinthus (1973), and Francis and Kannenberg (1978). For genotypic stability, Tai (1971), suggested partitioning the Genotype $x$ Environment interaction effects into " $\alpha$ ", which measures the linear response to environmental effects and " $\lambda$ " that describes the deviation from the linear response. Accordingly a stable genotype should have $\alpha=-1$ and $\lambda=1$. These measures have often been used in various studies to identify genotypes with better stability to be integrated into breeding programs of different crop species. 
This study aimed to identify promising alfalfa genotypes among six introduced alfalfa genotypes in comparison to a local genotype for future breeding program. Evaluation was based on their forage production and forage quality employing different stability measures to study the stability performance of genotypes along their growing period.

\section{MATERIALS AND METHODS}

A field trial was conducted at Nubaria Agricultural Research Station which represent calcareous soil type at the North West Delta, during the successive seasons 2014/2015 and 2015/2016. The stability performance of six introduced alfalfa genotypes across different environments in comparison to the local cultivar Ismailia-1was investigated. The introduced genotypes were; Us Stone, MI reya, WL903, WLML9, WL1111 and WL625 HQ, imported form the USA. All plant material was obtained from the Forage Crops Research Department at the Agriculture Research Center, Cairo, Egypt.

The trails were established in 2014 on December $19^{\text {th }}$ and proceeded till 2016 in randomized complete block design, with four replicates. The main soil physical and chemical properties are presented in Table (1) and the meteorological data for the experimental site is summarized in Table (2). Seeds were hand drilled at the rate of $20 \mathrm{Kg} / \mathrm{fad}$. in rows of $20 \mathrm{~cm}$ apart and plot size was $12 \mathrm{~m}^{2}(3 \times 4)$. Seeds were inoculated, prior to seeding, with Rhizobium melilotii. A starter dose of nitrogen fertilizer of $20 \mathrm{~kg} / \mathrm{fad}$ was applied directly, after emergence. Fertilizers applied were

Table 1: Soil physical and chemical properties of Nubaria Agriculture Research Station Farm.

\begin{tabular}{|c|c|c|}
\hline \multirow{2}{*}{ Characteristics } & \multicolumn{2}{|c|}{ Soil depth } \\
\hline & $0-30 \mathrm{~cm}$ & $30-60 \mathrm{~cm}$ \\
\hline $\begin{array}{l}\text { Texture } \\
\mathrm{pH}\end{array}$ & $\begin{array}{c}\text { Sandy loam } \\
8.23\end{array}$ & $\begin{array}{c}\text { Sandy loam } \\
8.26\end{array}$ \\
\hline \multicolumn{3}{|l|}{ Soil past extract: } \\
\hline $\mathrm{EC}(\mathrm{dS} / \mathrm{m})$ & 1.89 & 2.17 \\
\hline \multicolumn{3}{|l|}{ Cations (meq/L) } \\
\hline $\mathrm{Ca}^{2+}$ & 6.55 & 5.37 \\
\hline $\mathrm{Mg}^{2+}$ & 1.92 & 1.69 \\
\hline $\mathrm{K}^{+}$ & 1.72 & 2.34 \\
\hline $\mathrm{Na}^{+}$ & 8.71 & 12.30 \\
\hline \multicolumn{3}{|l|}{ Anions (meq/L) } \\
\hline $\mathrm{CO}_{3}^{2-}$ & - & - \\
\hline $\mathrm{HCO}_{3}^{-}$ & 5.57 & 6.50 \\
\hline $\mathrm{CL}^{-}$ & 9.72 & 11.62 \\
\hline $\mathrm{SO}_{4}{ }^{2-}$ & 3.61 & 3.49 \\
\hline Total CaCO3 (\%) & 21.29 & 23.14 \\
\hline O.M (\%) & 0.52 & 0.37 \\
\hline C.E.C.(meq/100) & 11.02 & 11.88 \\
\hline Total N (\%) & 0.023 & 0.025 \\
\hline Available P(mg/Kg) & 16.03 & 10.53 \\
\hline Exchangeable $\mathrm{K}(\mathrm{mg} / \mathrm{Kg})$ & 112.6 & 88.3 \\
\hline
\end{tabular}

super-phosphate $(150 \mathrm{~kg} / \mathrm{fad}$.$) and potassium$ sulphate $(50 \mathrm{~kg} / \mathrm{fad})$ each year. The super-phosphate fertilizer was applied once prior to seeding .The amount of potassium sulphate was divided into three $1 / 10$ bloom stage of maturity or when crown shoots reached $4-5 \mathrm{~cm}$ in length. Eighteen cuts were obtained starting from April $15^{\text {th }}$ in 2015 with an interval of 30-35 days between cuts and the last cut was on December $18^{\text {th }}$ in 2016. Each cut was considered an environment different from the other cuts according to Nascimento et al. (2013). The studied characters were; the mean plant height $(\mathrm{cm})$, determined at harvest by averaging five measurements from each plot, and an average over all cuts was scored. Annual fresh forage yields $(\mathrm{kg})$ were obtained by harvesting and weighing the plot summed over cuts to give total seasonal production and were converted into t/ha. Dry matter percentage (\%) was determined from plot samples of about $250 \mathrm{~g}$ of fresh forage dried in air flow oven at $70^{\circ} \mathrm{C}$ for $48 \mathrm{hrs}$, averaged overall cuts. Annual dry forage yield ( $\mathrm{t} / \mathrm{ha}$ ) calculated as the fresh forage yield ( $\mathrm{t} / \mathrm{ha}$ ) $\mathrm{x}$ dry matter $\%$ for each cut and summed over cuts to. Protein percentage (\%) was determined using standard methods (A.O.A.C., 1990). Annual protein yield $(\mathrm{kg} / \mathrm{ha})$ was calculated as the dry yield $(\mathrm{kg} / \mathrm{ha})$ $\mathrm{x}$ protein percentage $(\%)$ for each cut and summed over cuts. Fiber percentage (\%) was determined using standard methods (A.O.A.C., 1990), while annual fiber yield $(\mathrm{kg} / \mathrm{ha})$ was estimated dry yield $(\mathrm{kg} / \mathrm{ha}) \mathrm{x}$ fiber percentage $(\%)$ for each cut and summed over cuts. doses. Annually, alfalfa cultivars were harvested at 
Table 2: Number and date of alfalfa cuts and corresponding meteorological data at the experimental site.

\begin{tabular}{lccccccc}
\hline \multirow{2}{*}{$\begin{array}{l}\text { Cutting } \\
\text { noumber }\end{array}$} & \multirow{2}{*}{ Date of cut } & \multicolumn{3}{c}{ Air temp. } & \multicolumn{3}{c}{ Soil temp. } \\
\cline { 3 - 7 } Cut1 & $15-4-2015$ & 23.00 & 18.89 & 16.13 & 21.41 & 16.43 & 19.36 \\
Cut2 & $15-5-2015$ & 26.16 & 16.30 & 20.12 & 25.07 & 22.28 & 24.03 \\
Cut3 & $13-6-2015$ & 27.29 & 19.09 & 23.01 & 29.29 & 24.43 & 27.13 \\
Cut4 & $13-7-2015$ & 27.30 & 23.22 & 24.96 & 31.22 & 28.75 & 29.77 \\
Cut5 & $15-8-2015$ & 28.65 & 24.80 & 26.99 & 31.38 & 31.40 & 31.30 \\
Cut6 & $20-9-2015$ & 27.35 & 24.68 & 25.81 & 31.78 & 28.20 & 30.10 \\
Cut7 & $30-10-2015$ & 27.03 & 19.03 & 22.35 & 30.25 & 20.50 & 25.13 \\
Cut8 & $20-12-2015$ & 19.14 & 11.85 & 15.14 & 20.03 & 11.65 & 15.84 \\
Cut9 & $12-2-2016$ & 15.85 & 5.76 & 11.69 & 15.65 & 6.02 & 11.46 \\
Cut10 & $18-3-2016$ & 18.78 & 9.20 & 13.94 & 19.27 & 8.98 & 14.65 \\
Cut11 & $29-4-2016$ & 23.98 & 12.92 & 17.41 & 25.37 & 15.73 & 21.36 \\
Cut12 & $28-5-2016$ & 26.81 & 19.00 & 21.96 & 29.73 & 23.97 & 26.79 \\
Cut13 & $24-6-2016$ & 26.69 & 22.42 & 24.85 & 31.91 & 27.24 & 29.77 \\
Cut14 & $16-7-2016$ & 28.51 & 25.88 & 27.13 & 32.79 & 30.66 & 31.69 \\
Cut15 & $13-8-2016$ & 29.26 & 26.76 & 28.01 & 34.05 & 32.13 & 32.95 \\
Cut16 & $24-9-2016$ & 29.41 & 23.78 & 26.19 & 33.37 & 27.71 & 30.44 \\
Cut17 & $4-11-2016$ & 25.35 & 20.48 & 22.90 & 28.04 & 22.23 & 25.48 \\
Cut18 & $18-12-2016$ & 23.98 & 12.31 & 17.44 & 24.52 & 13.72 & 17.81 \\
\hline
\end{tabular}

\section{Statistical analysis:}

A regular analysis of variance was applied on individual environment, as indicated by Snedecor and Cochran (1989). Combined analysis of variance was performed on seven varieties over eighteen cutting dates (environments) to estimate the effects of genotype $\mathrm{x}$ environment interactions on the yielding ability when the assumption of homogeneity of variance can't be rejected. Genotypes were considered fixed, while, cutting dates (environments) were considered random variables. The processes of the analyses were carried out, using SAS program (SAS Institute. 2014).

Stability analysis:

Genotypic stability was measured according to the method described by Tai (1971). Parametric phenotypic stability on the other hand was estimated using eight different measure namely; Wricke (1962), Eberhart and Russell (1966) and Shukla (1972), Lin and Binns (1988) and non-parametric stability according to Nassar and Huehn (1987). The analysis was done by using Gen stat version $18^{\text {th }}$ edition software (VSN International, 2015).

\section{RESULTS AND DISCUSION}

\section{Analysis of variance}

Analysis of variance for the attributes studied (Table 3), have indicated highly significant differences among genotypes and among environments (cutting dates) for all studies traits. Genotype $\mathrm{x}$ environment interactions were highly significant for all traits with the exception of plant height.

\section{1- Plant height:}

Mean plant height for the seven tested varieties of alfalfa under different environments was presented in Table (4). The local variety Ismailia-1 showed the highest significant value for average plant height $(59.53 \mathrm{~cm})$ compared to the exotic varieties. The varieties WL625HQ, Us stone, WL1111, MI reya and WL903, on the other hand gave the least average plant height of 55.78, 55.90, $56.47,56.76$ and $57.28 \mathrm{~cm}$, respectively. The effects of cutting date (environments), were significant where the environments E11 and E12 (April and May 2016) gave the highest significant average plant height (70.57 and $70.71 \mathrm{~cm}$, respectively), with no significant differences between those two environments. The least average plant height (42.61 $\mathrm{cm}$ ) was recorded for environment E9 (February 2016). In general, the average plant height across the tested varieties was higher in spring followed by summer. The winter months gave the least average plant height (Table 4). These results in agreement with those reported by Rammah et al. (1995) and Oushy et al. (2007).

\section{2- Fresh and dry forage yield:}

Both fresh and dry forage yields for the seven tested varieties of alfalfa under different environments were presented in Table (5) and Table (6), respectively. In general, the local cultivar, Ismailia-1, significantly outperformed all exotic genotypes recording the highest average fresh and dry forage yield over environments (11.10 t/ha and $2.243 \mathrm{t} / \mathrm{ha}$, respectively). 


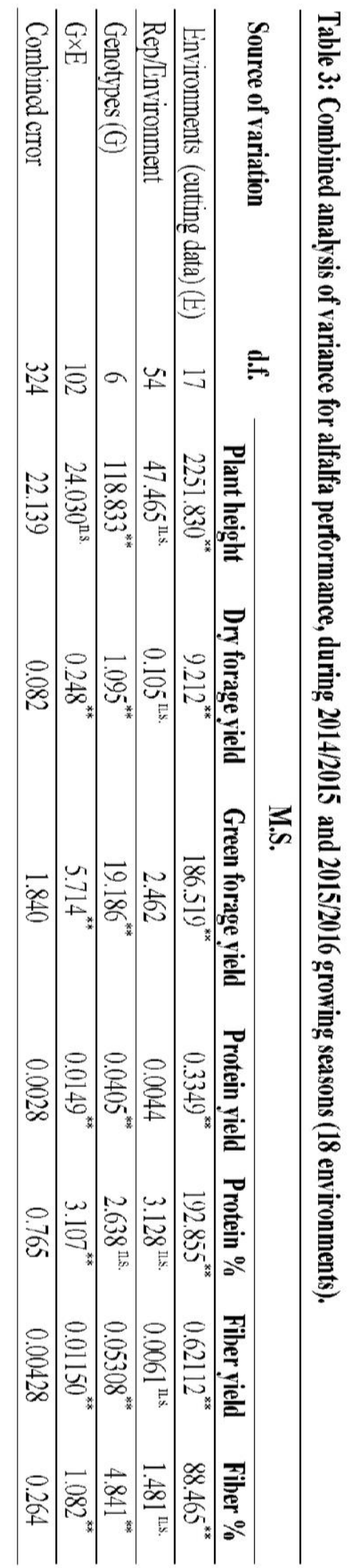


Table 4: Mean performance of alfalfa genotypes for plant height $(\mathrm{cm})$ over the 18 studied cuttings.

\begin{tabular}{cccccccccc}
\hline $\begin{array}{c}\text { Environment } \\
\text { (E) }\end{array}$ & \multirow{2}{*}{$\begin{array}{c}\text { Cutting } \\
\text { date }\end{array}$} & $\begin{array}{c}\text { Us } \\
\text { Stone }\end{array}$ & MI reya & WL903 & WLML9 & WL1111 & WL625HQ & Ismailia-1 & Mean \\
\hline E1 & Cut1 & 46.75 & 45.50 & 50.25 & 46.25 & 44.25 & 43.75 & 46.50 & 46.179 \\
\hline E2 & Cut2 & 45.75 & 46.25 & 51.25 & 47.50 & 44.00 & 44.50 & 48.75 & 46.857 \\
\hline E3 & Cut3 & 64.50 & 64.00 & 62.75 & 63.00 & 64.25 & 62.75 & 68.50 & 64.250 \\
\hline E4 & Cut4 & 66.75 & 59.25 & 63.50 & 65.25 & 69.00 & 67.50 & 71.25 & 66.250 \\
\hline E5 & Cut5 & 61.75 & 61.00 & 64.50 & 60.50 & 62.00 & 59.50 & 65.50 & 62.107 \\
\hline E6 & Cut6 & 50.00 & 52.75 & 52.00 & 52.50 & 49.50 & 53.00 & 53.25 & 51.857 \\
\hline E7 & Cut7 & 48.00 & 49.25 & 49.00 & 49.50 & 46.50 & 49.75 & 50.25 & 48.893 \\
\hline E8 & Cut8 & 50.25 & 51.75 & 54.00 & 53.25 & 52.00 & 51.00 & 54.75 & 52.429 \\
\hline E9 & Cut9 & 39.75 & 47.75 & 43.00 & 44.50 & 39.75 & 38.50 & 45.00 & 42.607 \\
\hline E10 & Cut10 & 44.25 & 49.25 & 48.50 & 48.50 & 43.50 & 44.00 & 55.75 & 47.679 \\
\hline E11 & Cut11 & 70.25 & 70.00 & 66.25 & 67.00 & 68.50 & 71.25 & 80.75 & 70.571 \\
\hline E12 & Cut12 & 69.25 & 67.50 & 72.00 & 73.75 & 71.00 & 68.25 & 73.50 & 70.714 \\
\hline E13 & Cut13 & 66.00 & 68.75 & 66.25 & 69.50 & 68.25 & 66.00 & 66.50 & 67.643 \\
\hline E14 & Cut14 & 61.75 & 67.50 & 65.50 & 66.50 & 65.00 & 63.50 & 69.00 & 65.536 \\
\hline E15 & Cut15 & 55.25 & 52.00 & 54.00 & 54.00 & 55.75 & 54.25 & 52.00 & 53.893 \\
\hline E16 & Cut16 & 53.00 & 55.50 & 52.00 & 59.00 & 56.25 & 54.25 & 58.50 & 55.500 \\
\hline E17 & Cut17 & 51.75 & 55.00 & 57.75 & 57.00 & 58.25 & 54.50 & 53.00 & 55.321 \\
\hline E18 & Cut18 & 59.00 & 59.00 & 58.50 & 60.25 & 59.00 & 57.75 & 57.50 & 58.679 \\
\hline Mean & & 55.903 & 56.764 & 57.278 & 57.653 & 56.472 & 55.778 & 59.528 & 57.054 \\
\hline L.S.D0.05 Genotypes =1.577 & & & & & & & & \\
\hline L.S.D 0.05 Env. $=$ & 2.563 & & & & & & & & \\
\hline L.S.D 0.05 GxEnv. $=$ & n.s & & & & & & & & \\
\hline
\end{tabular}

Table 5: Mean performance of alfalfa genotypes as green forage yield over the 18 cuttings (environments)

\begin{tabular}{cccccccccc}
\hline $\begin{array}{c}\text { Environment } \\
(\text { E) }\end{array}$ & $\begin{array}{c}\text { Cut } \\
\text { date }\end{array}$ & Us Stone & MI reya & WL903 & WLML9 & WL1111 & WL625HQ & Ismailia-1 & Mean \\
\hline E1 & Cut1 & 3.990 & 6.840 & 5.640 & 6.960 & 6.450 & 5.385 & 7.020 & 6.040 \\
\hline E2 & Cut2 & 14.900 & 12.100 & 10.700 & 9.350 & 14.500 & 9.375 & 10.550 & 11.639 \\
\hline E3 & Cut3 & 16.200 & 13.500 & 13.350 & 12.750 & 13.500 & 12.000 & 12.750 & 13.435 \\
\hline E4 & Cut4 & 10.435 & 10.465 & 10.215 & 9.210 & 10.420 & 10.810 & 13.050 & 10.657 \\
\hline E5 & Cut5 & 9.120 & 9.170 & 8.015 & 9.090 & 8.760 & 9.810 & 12.200 & 9.452 \\
\hline E6 & Cut6 & 11.450 & 11.275 & 10.660 & 11.650 & 10.975 & 10.775 & 11.060 & 11.120 \\
\hline E7 & Cut7 & 10.325 & 10.170 & 9.250 & 10.310 & 9.800 & 9.570 & 9.650 & 9.867 \\
\hline E8 & Cut8 & 6.600 & 6.750 & 6.600 & 5.950 & 8.803 & 6.903 & 9.450 & 7.293 \\
\hline E9 & Cut9 & 4.640 & 6.300 & 4.950 & 5.580 & 4.870 & 4.124 & 8.450 & 5.560 \\
\hline E10 & Cut10 & 5.700 & 8.250 & 7.500 & 7.680 & 7.950 & 6.450 & 12.825 & 8.050 \\
\hline E11 & Cut11 & 9.600 & 11.350 & 10.200 & 11.925 & 6.900 & 8.730 & 14.250 & 10.422 \\
\hline E12 & Cut12 & 15.300 & 15.350 & 13.550 & 14.450 & 13.950 & 14.250 & 15.725 & 14.653 \\
\hline E13 & Cut13 & 15.600 & 13.200 & 15.350 & 13.500 & 14.700 & 15.400 & 15.025 & 14.682 \\
\hline E14 & Cut14 & 10.250 & 9.650 & 10.275 & 9.630 & 10.315 & 10.100 & 10.490 & 10.101 \\
\hline E15 & Cut15 & 11.000 & 10.390 & 11.275 & 10.575 & 11.250 & 11.075 & 11.475 & 11.005 \\
\hline E16 & Cut16 & 9.910 & 8.690 & 9.030 & 9.113 & 7.890 & 8.967 & 8.935 & 8.933 \\
\hline E17 & Cut17 & 10.000 & 10.560 & 9.730 & 9.380 & 9.535 & 9.930 & 8.460 & 9.656 \\
\hline E18 & Cut18 & 8.005 & 7.400 & 7.750 & 8.340 & 7.625 & 8.270 & 8.230 & 7.945 \\
\hline Mean & & 10.168 & 10.078 & 9.668 & 9.746 & 9.899 & 9.551 & 11.088 & 10.028 \\
\hline L.S.D0.05 bet. Genotypes $=0.724$ & & & & & & & & & \\
\hline L.S.D0.05 bet. Env. = 0.796 & & & & & & & & & \\
\hline L.S.D0.05 GxEnv. = 1.918 & & & & & & & & & \\
\hline
\end{tabular}


The least yielding genotype was the genotype WL625HQ, showing an average fresh forage yield of $9.55 \mathrm{t} / \mathrm{ha}$ and an average dry forage yield of 1.86 $\mathrm{t} /$ ha (Table 5). Because the interaction between cutting dates and genotype was significant, it was possible to compare genotypes under different cutting dates. The highest fresh forage yield (16.0 $\mathrm{t} / \mathrm{ha}$ ) was recorded for the genotype US-Stone cut in June-2015 (cut3), however this value did not significantly differ from cuts taken from the same genotype in May-2015 (cut2), May-2016 (cut12), June-2016 (cut13) or the genotype MI reya, WLML9, Ismailia-1 cut in May-2016 (cut12) and the genotypes WL903m, WL1111, WL625HQ and Ismailia-1 cut on June-2016 (cut13) as seen in Table (5). The least fresh forage yield values were recorded for the genotypes; US stone, WL903 and WL625HQ, either cut in the April-2015 (cut1) or February-2016 (cut9) and the genotype WLML9 cut in December-2015 (cut8) or in February-2016 (cut9).

Observing the dry forage yield in Table (6) indicated that the highest value (3.43 t/ha) was recorded for the genotype US-stone cut in May2016 (cut12). This value did not differ significantly from that observed for the same genotype in May2015 (cut2), or the genotypes MI reya, WL111, WL625HQ and Ismailia-1 cut in May-2016 (cut12) and that of the genotypes MI reya, WL903, WL625HQ and Ismailia-1 cut in June-2016 (cut13). The least value for the dry forage yield $(0.68 \mathrm{t} / \mathrm{ha})$ was recorded for the US stone cut in April-2015 (cut1) and that value did not differ significantly from the values for the same genotype cut in December, February and March-2106 (cuts 8, 9 and 10 , respectively). At the same time these values did not significantly differ from the values obtained for the genotype WL903 or WL635HQ in cut9 as indicated in Table (6).

The genotype US-Stone seemes to be quite different from all tested genotypes where it produces it maximum forage yield (fresh and dry) very early after plant establishment (cuts 2 and 3) and delivers high yields once again in cut 12, as most genotypes did. Although, US Stone ranks second in both dry and fresh yield after the local cultivar Ismailia-1, the distribution of forage production along the 18 environments experienced high fluctuations compared to Ismailia-1 that presented stability and continuity in forage production along the environments (Table 5 and 6). These results are in full agreement with Abdel-Galil and Hamed (2008), who indicated that the cultivar Ismailia-1 expressed significant performance and wide range of adaptability compared to exotic cultivars.

Table 6: Mean performance of alfalfa genotypes for dry forage yield over the 18 cuttings (environments).

\begin{tabular}{|c|c|c|c|c|c|c|c|c|c|}
\hline \multirow{2}{*}{$\begin{array}{c}\text { Environment } \\
\text { (E) }\end{array}$} & \multirow{2}{*}{ Cut date } & \multirow[b]{2}{*}{ Us Stone } & \multirow[b]{2}{*}{ MI reya } & \multirow[b]{2}{*}{ WL903 } & \multicolumn{5}{|c|}{ Genotypes } \\
\hline & & & & & WLML9 & WL1111 & WL625HQ & Ismailia-1 & Mean \\
\hline E1 & Cut1 & 0.677 & 1.233 & 1.106 & 1.330 & 1.075 & 0.905 & 1.189 & 1.073 \\
\hline E2 & Cut2 & 3.129 & 2.424 & 1.810 & 1.815 & 2.687 & 1.868 & 2.083 & 2.259 \\
\hline E3 & Cut3 & 2.838 & 2.129 & 2.254 & 2.451 & 2.542 & 2.230 & 2.548 & 2.442 \\
\hline E4 & Cut4 & 2.224 & 2.128 & 2.148 & 1.958 & 2.193 & 2.207 & 2.707 & 2.223 \\
\hline E5 & Cut5 & 1.914 & 2.008 & 1.550 & 2.024 & 1.928 & 1.732 & 2.526 & 1.954 \\
\hline E6 & Cut6 & 2.312 & 2.170 & 1.865 & 2.045 & 2.107 & 1.770 & 2.134 & 2.057 \\
\hline E7 & Cut7 & 1.998 & 1.872 & 1.545 & 2.063 & 1.756 & 1.569 & 1.801 & 1.801 \\
\hline E8 & Cut8 & 1.070 & 1.181 & 1.180 & 1.098 & 1.620 & 1.220 & 1.587 & 1.279 \\
\hline E9 & Cut9 & 0.929 & 1.353 & 0.987 & 1.141 & 0.956 & 0.783 & 1.743 & 1.127 \\
\hline E10 & Cut10 & 0.975 & 1.488 & 1.471 & 1.484 & 1.305 & 1.075 & 2.176 & 1.427 \\
\hline E11 & Cut11 & 2.244 & 2.689 & 2.425 & 2.296 & 1.541 & 2.070 & 3.226 & 2.356 \\
\hline E12 & Cut12 & 3.427 & 3.141 & 2.918 & 3.117 & 2.678 & 3.192 & 3.251 & 3.103 \\
\hline E13 & Cut13 & 2.868 & 2.967 & 3.162 & 2.714 & 2.786 & 3.150 & 2.983 & 2.947 \\
\hline E14 & Cut14 & 2.115 & 2.134 & 2.304 & 2.160 & 2.334 & 2.098 & 2.335 & 2.212 \\
\hline E15 & Cut15 & 2.492 & 2.363 & 2.619 & 2.299 & 2.350 & 2.319 & 2.573 & 2.413 \\
\hline E16 & Cut16 & 2.163 & 1.860 & 1.946 & 1.931 & 1.642 & 1.734 & 1.975 & 1.893 \\
\hline E17 & Cut17 & 2.031 & 2.169 & 2.034 & 1.902 & 1.965 & 2.014 & 1.893 & 2.001 \\
\hline E18 & Cut18 & 1.347 & 1.339 & 1.411 & 1.612 & 1.443 & 1.448 & 1.653 & 1.465 \\
\hline Mean & & 2.042 & 2.036 & 1.930 & 1.969 & 1.940 & 1.860 & 2.243 & 2.003 \\
\hline \multicolumn{10}{|c|}{ L.S.D 0.05 Genotypes $=0.153$} \\
\hline \multicolumn{10}{|c|}{ L.S.D 0.05 Env. $=0.165$} \\
\hline \multicolumn{10}{|c|}{ L.S.D ${ }_{0.05}$ GxEnv. $=0.404$} \\
\hline
\end{tabular}




\section{3- Protein yield and protein percentage:}

The highest protein percentage was exhibited by MI-reya, where, it gave $19.38 \%$ (Table7). The least protein content was US-Stone, which gave $18.093 \%$. It was valuable to note that, the highest percentage of protein matched with harvests, which corresponded to low temperature. Contrary to the productivity, which increased with the increase in temperature. The highest protein percentage were provided by WL1111, WL 625HQ in December2015 (cut 8), where it was 23.98 and 23.71, respectively. The least protein percentage was resulted from Ismailia-1 in cut $5(13.04 \%)$.

Total protein yield for seven tested varieties of alfalfa under different environments were presented in (Table 8). Ismailia-1recorded the highest protein yield over environments (0.409 t/ha) with insignificantly difference from the variety MI reya (0.392 t/ha). While, the variety WL625HQ gave the least forage protein yield (0.339 t/ha).

Significant high protein yield were obtained under the $12^{\text {th }}$ cutting (May-2016) (0.641 t/ha), Followed significantly by the $13^{\text {th }}$ and $11^{\text {th }}$ cuttings (June and April-2016) (0.528 and 0.514 t/ha). The least protein yield $(0.183 \mathrm{t} / \mathrm{ha})$ obtained from first cutting (April-2015).

The highest protein yield obtained from Ismailia-1 (0.761t/ha) under $11^{\text {th }}$ cutting (April-
2016) insignificantly by Ismailia-1 and Us. Sotne in $12^{\text {th }}$ cutting (May-2016) (0.722 and $0.726 \mathrm{t} / \mathrm{ha}$ respectively). While, first cutting the variety Us Stone gave the least protein yield $(0.106 \mathrm{t} / \mathrm{ha})$ followed in significant by WL625HQ (0.146 t/ha).

\section{4- Fiber yield and fiber percentage:}

Average of fiber percentage of the 18 environments (Table 9). MI reya (22.96\%). Had significant similar fiber percentage as US. Stone $(22.645 \%)$. The least fiber percentage provided by Ismailia-1, which (22.08\%). The highest percentage of fibers was recorded for US.Stone in $14^{\text {th }}$ cutting, $(26.53 \%) 15^{\text {th }}$ cutting, $(26.43 \%)$. The MI reya showed fiber percentages $26.18 \%$, at cutting 15 and 14 , respectively, percentage of the fibers obtained from Ismailia-1 in $9^{\text {th }}$ cutting, (19.56\%).

Fiber yield for seven tested varieties of alfalfa under different environments were presented in (Table 10). Ismailia-1 recorded the highest fiber yield over environments (0.498 t/ha) followed significantly MI reya $(0.473 \mathrm{t} / \mathrm{ha})$. The variety WL625HQ in gave the least fiber yield $(0.416 \mathrm{t} / \mathrm{ha})$. The highest fiber yield were obtained under the $13^{\text {th }}$ cutting (April 2016 (0.721 t/ha), Followed in significantly by the $12^{\text {th }}$ cutting (May 2016) $(0.708$ $\mathrm{t} / \mathrm{ha})$. The least fiber yield $(0.222 \mathrm{t} / \mathrm{ha})$ was obtained from the $9^{\text {th }}$ cutting (Fib.-2016).

Table 7: Mean performance of alfalfa genotypes for protein percentage over the 18 cuttings (environments).

\begin{tabular}{cccccccccc}
\hline $\begin{array}{c}\text { Environment } \\
(\text { E) }\end{array}$ & $\begin{array}{c}\text { Cutting } \\
\text { date }\end{array}$ & Us Stone & MI reya & WL903 & WLML9 & WL1111 & WL625HQ & Ismailia-1 & Mean \\
\cline { 3 - 10 } E1 & Cut1 & 15.700 & 17.582 & 17.112 & 16.690 & 18.695 & 16.137 & 17.082 & 16.999 \\
\hline E2 & Cut2 & 17.690 & 18.885 & 16.922 & 17.382 & 20.690 & 16.530 & 16.610 & 17.815 \\
\hline E3 & Cut3 & 15.330 & 17.435 & 15.222 & 13.827 & 15.805 & 16.212 & 15.735 & 15.652 \\
\hline E4 & Cut4 & 14.172 & 16.245 & 13.905 & 13.157 & 14.900 & 16.330 & 16.095 & 14.972 \\
\hline E5 & Cut5 & 13.942 & 14.665 & 13.752 & 13.067 & 13.425 & 13.702 & 13.080 & 13.661 \\
\hline E6 & Cut6 & 16.110 & 17.700 & 16.187 & 16.750 & 14.767 & 16.712 & 16.107 & 16.333 \\
\hline E7 & Cut7 & 17.550 & 19.797 & 18.642 & 20.757 & 17.557 & 18.682 & 17.595 & 18.652 \\
\hline E8 & Cut8 & 21.230 & 21.765 & 19.605 & 22.945 & 23.850 & 23.605 & 22.965 & 22.280 \\
\hline E9 & Cut9 & 20.315 & 23.230 & 20.320 & 22.110 & 23.195 & 22.722 & 21.285 & 21.882 \\
\hline E10 & Cut10 & 19.662 & 22.735 & 20.167 & 21.287 & 22.247 & 22.135 & 21.057 & 21.327 \\
\hline E11 & Cut11 & 20.077 & 21.277 & 22.340 & 20.145 & 21.787 & 23.100 & 23.655 & 21.768 \\
\hline E12 & Cut12 & 21.267 & 21.787 & 20.875 & 19.822 & 19.172 & 19.220 & 22.252 & 20.627 \\
\hline E13 & Cut13 & 18.222 & 19.522 & 19.775 & 17.685 & 16.907 & 17.742 & 15.540 & 17.913 \\
\hline E14 & Cut14 & 16.167 & 17.357 & 18.800 & 17.147 & 17.675 & 15.902 & 16.637 & 17.097 \\
\hline E15 & Cut15 & 18.075 & 16.925 & 18.710 & 17.240 & 18.607 & 17.140 & 16.235 & 17.561 \\
\hline E16 & Cut16 & 19.242 & 19.220 & 20.132 & 18.775 & 19.260 & 17.675 & 17.157 & 18.780 \\
\hline E17 & Cut17 & 18.805 & 20.682 & 21.160 & 22.320 & 19.557 & 18.845 & 18.332 & 19.957 \\
\hline E18 & Cut18 & 22.115 & 22.047 & 21.425 & 21.310 & 22.022 & 22.157 & 22.287 & 21.909 \\
\hline Mean & & 18.093 & 19.381 & 18.614 & 18.467 & 18.895 & 18.586 & 18.317 & 18.622 \\
\hline ES Do & & & & & & & &
\end{tabular}

L.S.D 0.05 Genotypes $=0.575$

L.S.D 0.05 Env. $=0.457$

L.S.D 0.05 GxEnv. $=1.210$ 
Table 8: Mean performance of alfalfa genotypes for protein yield (t/ha) over the 18 cuttings (environments).

\begin{tabular}{|c|c|c|c|c|c|c|c|c|c|}
\hline \multirow{2}{*}{$\begin{array}{l}\text { Environment } \\
\text { (E) }\end{array}$} & \multirow{2}{*}{$\begin{array}{c}\text { Cutting } \\
\text { date }\end{array}$} & \multicolumn{8}{|c|}{ Genotypes } \\
\hline & & Us Stone & MI reya & WL903 & WLML9 & WL1111 & WL625HQ & Ismailia-1 & Mean \\
\hline E1 & Cut1 & 0.106 & 0.216 & 0.189 & 0.222 & 0.201 & 0.146 & 0.203 & 0.183 \\
\hline E2 & Cut2 & 0.554 & 0.457 & 0.307 & 0.299 & 0.556 & 0.310 & 0.345 & 0.404 \\
\hline E3 & Cut3 & 0.436 & 0.375 & 0.344 & 0.337 & 0.401 & 0.378 & 0.399 & 0.381 \\
\hline E4 & Cut4 & 0.315 & 0.346 & 0.297 & 0.257 & 0.325 & 0.360 & 0.435 & 0.334 \\
\hline E5 & Cut5 & 0.267 & 0.293 & 0.212 & 0.264 & 0.259 & 0.238 & 0.330 & 0.266 \\
\hline E6 & Cut6 & 0.372 & 0.382 & 0.301 & 0.343 & 0.311 & 0.295 & 0.344 & 0.336 \\
\hline E7 & Cut7 & 0.351 & 0.371 & 0.288 & 0.429 & 0.308 & 0.293 & 0.317 & 0.337 \\
\hline E8 & Cut8 & 0.226 & 0.257 & 0.231 & 0.251 & 0.386 & 0.288 & 0.363 & 0.286 \\
\hline E9 & Cut9 & 0.188 & 0.313 & 0.200 & 0.252 & 0.222 & 0.178 & 0.371 & 0.246 \\
\hline E10 & Cut10 & 0.191 & 0.338 & 0.296 & 0.315 & 0.294 & 0.238 & 0.458 & 0.304 \\
\hline E11 & Cut11 & 0.450 & 0.571 & 0.539 & 0.462 & 0.334 & 0.478 & 0.761 & 0.514 \\
\hline E12 & Cut12 & 0.726 & 0.684 & 0.610 & 0.617 & 0.515 & 0.613 & 0.722 & 0.641 \\
\hline E13 & Cut13 & 0.522 & 0.584 & 0.622 & 0.478 & 0.470 & 0.559 & 0.463 & 0.528 \\
\hline E14 & Cut14 & 0.341 & 0.370 & 0.432 & 0.370 & 0.412 & 0.333 & 0.388 & 0.378 \\
\hline E15 & Cut15 & 0.450 & 0.400 & 0.490 & 0.396 & 0.436 & 0.397 & 0.417 & 0.427 \\
\hline E16 & Cut16 & 0.416 & 0.357 & 0.391 & 0.363 & 0.316 & 0.306 & 0.338 & 0.355 \\
\hline E17 & Cut17 & 0.381 & 0.448 & 0.431 & 0.424 & 0.384 & 0.379 & 0.346 & 0.399 \\
\hline E18 & Cut18 & 0.298 & 0.295 & 0.303 & 0.343 & 0.317 & 0.320 & 0.368 & 0.321 \\
\hline Mean & & 0.366 & 0.392 & 0.360 & 0.357 & 0.358 & 0.339 & 0.409 & 0.369 \\
\hline \multicolumn{10}{|c|}{ L.S.D $D_{0.05}$ Genotypes $=0.0283$} \\
\hline \multicolumn{10}{|c|}{ L.S.D 0.05 Env. $=0.0407$} \\
\hline \multicolumn{10}{|c|}{ L.S.D 0.05 GxEnv. $=0.0720$} \\
\hline
\end{tabular}

Table 9: Mean performance of alfalfa genotypes for fiber percentage over the 18 cuttings (environments).

\begin{tabular}{|c|c|c|c|c|c|c|c|c|c|}
\hline \multirow{2}{*}{$\begin{array}{l}\text { Environment } \\
\text { (E) }\end{array}$} & \multirow{2}{*}{$\begin{array}{c}\text { Cutting } \\
\text { date }\end{array}$} & \multicolumn{8}{|c|}{ Genotypes } \\
\hline & & Us Stone & MI reya & WL903 & WLML9 & WL1111 & WL625HQ & Ismailia-1 & Mean \\
\hline E1 & Cut1 & 21.502 & 20.857 & 20.360 & 20.745 & 20.260 & 21.755 & 20.402 & 20.840 \\
\hline E2 & Cut2 & 20.895 & 19.960 & 20.860 & 21.860 & 22.290 & 22.325 & 20.767 & 21.279 \\
\hline E3 & Cut3 & 22.905 & 21.837 & 23.240 & 20.702 & 21.045 & 20.322 & 21.372 & 21.631 \\
\hline E4 & Cut4 & 23.240 & 23.307 & 23.235 & 22.217 & 22.130 & 21.725 & 22.167 & 22.574 \\
\hline E5 & Cut5 & 24.297 & 24.955 & 24.825 & 24.907 & 25.072 & 24.112 & 23.840 & 24.572 \\
\hline E6 & Cut6 & 22.240 & 23.207 & 22.045 & 21.145 & 23.242 & 22.025 & 22.422 & 22.332 \\
\hline E7 & Cut7 & 21.025 & 21.202 & 21.727 & 21.252 & 21.262 & 22.055 & 21.052 & 21.367 \\
\hline E8 & Cut8 & 20.242 & 20.200 & 21.270 & 21.110 & 20.125 & 20.042 & 19.212 & 20.314 \\
\hline E9 & Cut9 & 20.080 & 19.715 & 19.442 & 19.902 & 19.680 & 20.267 & 19.282 & 19.766 \\
\hline E10 & Cut10 & 20.707 & 21.080 & 20.305 & 20.122 & 20.457 & 20.510 & 20.135 & 20.473 \\
\hline E11 & Cut11 & 21.717 & 22.220 & 19.745 & 20.867 & 21.807 & 21.135 & 20.750 & 21.177 \\
\hline E12 & Cut12 & 22.312 & 23.762 & 23.315 & 23.375 & 23.270 & 22.300 & 21.750 & 22.869 \\
\hline E13 & Cut13 & 25.222 & 24.937 & 26.037 & 23.732 & 24.127 & 23.072 & 24.190 & 24.473 \\
\hline E14 & Cut14 & 26.262 & 26.217 & 25.205 & 25.840 & 25.080 & 24.345 & 25.052 & 25.428 \\
\hline E15 & Cut15 & 25.805 & 26.040 & 24.385 & 24.972 & 25.240 & 25.112 & 25.280 & 25.262 \\
\hline E16 & Cut16 & 24.042 & 25.325 & 24.190 & 25.247 & 24.192 & 24.547 & 25.047 & 24.655 \\
\hline E17 & Cut17 & 23.035 & 25.207 & 22.420 & 23.230 & 22.192 & 21.275 & 22.742 & 22.871 \\
\hline E18 & Cut18 & 22.085 & 23.327 & 22.852 & 22.370 & 22.050 & 22.330 & 22.100 & 22.444 \\
\hline Mean & & 22.645 & 22.964 & 22.525 & 22.422 & 22.418 & 22.180 & 22.087 & 22.463 \\
\hline \multicolumn{10}{|c|}{ L.S.D ${ }_{0.05}$ Genotypes $=0.339$} \\
\hline \multicolumn{10}{|c|}{ L.S.D 0.05 Env. $=0.268$} \\
\hline \multicolumn{10}{|c|}{ L.S.D0.05 GxEnv. $=0.711$} \\
\hline
\end{tabular}


Table 10: Mean performance of alfalfa genotypes for fiber yield (t/ha) over the 18 cuts studied.

\begin{tabular}{|c|c|c|c|c|c|c|c|c|c|}
\hline \multirow{2}{*}{$\begin{array}{c}\text { Environment } \\
\text { (E) } \\
\end{array}$} & \multirow{2}{*}{$\begin{array}{c}\text { Cutting } \\
\text { date }\end{array}$} & \multicolumn{8}{|c|}{ Genotypes } \\
\hline & & Us Stone & MI reya & WL903 & WLML9 & WL1111 & WL625HQ & Ismailia-1 & Mean \\
\hline E1 & Cut1 & 0.145 & 0.257 & 0.225 & 0.276 & 0.217 & 0.196 & 0.242 & 0.223 \\
\hline E2 & Cut2 & 0.653 & 0.483 & 0.377 & 0.396 & 0.599 & 0.415 & 0.432 & 0.480 \\
\hline E3 & Cut3 & 0.650 & 0.467 & 0.525 & 0.507 & 0.534 & 0.473 & 0.544 & 0.529 \\
\hline E4 & Cut4 & 0.517 & 0.497 & 0.499 & 0.434 & 0.485 & 0.478 & 0.599 & 0.501 \\
\hline E5 & Cut5 & 0.464 & 0.501 & 0.385 & 0.503 & 0.483 & 0.417 & 0.601 & 0.479 \\
\hline E6 & Cut6 & 0.514 & 0.503 & 0.411 & 0.432 & 0.490 & 0.389 & 0.478 & 0.460 \\
\hline E7 & Cut7 & 0.420 & 0.396 & 0.335 & 0.439 & 0.373 & 0.346 & 0.379 & 0.384 \\
\hline E8 & Cut8 & 0.216 & 0.238 & 0.251 & 0.231 & 0.326 & 0.244 & 0.304 & 0.259 \\
\hline E9 & Cut9 & 0.186 & 0.266 & 0.191 & 0.226 & 0.188 & 0.159 & 0.335 & 0.222 \\
\hline E10 & Cut10 & 0.201 & 0.313 & 0.298 & 0.298 & 0.270 & 0.221 & 0.438 & 0.291 \\
\hline E11 & Cut11 & 0.487 & 0.597 & 0.478 & 0.478 & 0.334 & 0.437 & 0.668 & 0.497 \\
\hline E12 & Cut12 & 0.764 & 0.746 & 0.679 & 0.727 & 0.623 & 0.711 & 0.706 & 0.708 \\
\hline E13 & Cut13 & 0.723 & 0.739 & 0.823 & 0.644 & 0.672 & 0.726 & 0.721 & 0.721 \\
\hline E14 & Cut14 & 0.555 & 0.559 & 0.580 & 0.558 & 0.585 & 0.510 & 0.585 & 0.562 \\
\hline E15 & Cut15 & 0.642 & 0.615 & 0.638 & 0.574 & 0.593 & 0.582 & 0.650 & 0.613 \\
\hline E16 & Cut16 & 0.520 & 0.472 & 0.470 & 0.488 & 0.397 & 0.425 & 0.494 & 0.466 \\
\hline E17 & Cut17 & 0.467 & 0.547 & 0.456 & 0.442 & 0.436 & 0.428 & 0.430 & 0.458 \\
\hline E18 & Cut18 & 0.297 & 0.312 & 0.323 & 0.360 & 0.318 & 0.323 & 0.365 & 0.328 \\
\hline Mean & & 0.468 & 0.473 & 0.441 & 0.445 & 0.440 & 0.416 & 0.498 & 0.4549 \\
\hline \multicolumn{10}{|c|}{ L.S.D $0.05 \quad$ Genotypes $=0.0349$} \\
\hline \multicolumn{10}{|c|}{ L.S.D 0.05 Env. $=0.0357$} \\
\hline L.S.D ${ }_{0.05} \quad \mathrm{Gx}$ & $=0.0$ & & & & & & & & \\
\hline
\end{tabular}

WL903 gave the highest fiber yield (0.823t/ha) under the $13^{\text {th }}$ cutting (June-2016) followed insignificantly by Us Stone and MI reya at the $12^{\text {th }}$ cutting (0.764 and $0.746 \mathrm{t} / \mathrm{ha})$ and MI reya in $13^{\text {th }}$ cutting $(0.739 \mathrm{t} / \mathrm{ha})$. While Us Stone in $1^{\text {st }}$ cutting recorded the least fiber yield (0.0.145 t/ha).

In the first year of experiment a lower content of crude protein was obtained compared to second year for the different harvest stages, because in the first year of the experiment the alfalfa is installing for yield potentiality and most of the nitrogen is used for this. The same effect was pointed out by Decmyenaere et al., (2008) and Stanacev et al., 2010. According to the harvest stage it was observed a reduction of $\mathrm{CP}$ and an increase in $\mathrm{CF}$ in the late harvest which could be explained by the evolution of stems and leaves containing more CP and less CF than stems. Moreover Heinriches, 1970, and Babinec et al., 2001, pointed- out that, losses of leaves are important because the protein concentration was higher in leaves than in stems. The crude protein content and the crude fiber contents vary between very wide limits depending largely on the development stage alfalfa (Dale, 2011). Crud fiber concentration varied in response to the growing season. Spring and summer growth had higher crude fiber concentration than winter and autumn (Abd El-Halim et al. 1992).

Stability analysis:

Combined analysis over environments for fresh and dry forage yield, protein $\%$ and fiber $\%$ of seven varieties of alfalfa were presented in Table 11. Analysis of variance across genotypes and environments showed that mean squares due to genotypes were highly significant for all traits except for protein \%. The environment effect represented a highly significant mean squares for all traits. The environment $\mathrm{x}$ genotype interaction was only significant for fresh and dry forage yield, (Protein $\%$ and fiber $\%$ were not significant).

Table 11: combined analysis over environments for fresh and dry forage yields, protein \% and fiber\% of seven varieties of alfalfa.

\begin{tabular}{lccccc}
\hline \multirow{2}{*}{ Source of variation } & \multirow{2}{*}{ d.f. } & \multicolumn{3}{c}{ M.S. } \\
\cline { 3 - 6 } & 125 & Green forage yield & dry forage yield & Protein \% & Fiber \% \\
\hline Total & 6 & 7.737 & 0.3801 & 7.222 & 3.2869 \\
\hline Genotypes & 119 & $7.795^{* *}$ & $0.2688^{* *}$ & 0.653 & $1.2148^{* * *}$ \\
Env +Gen. * Env. & 1 & $792.703^{* *}$ & 0.3857 & 7.553 & 3.3914 \\
\hline Env (linear). & 6 & $3.807^{*}$ & $39.286^{* *}$ & $819.600^{* *}$ & $376.015^{* *}$ \\
\hline Gen*Env (linear). & 112 & 1.097 & $0.1928^{*}$ & 0.609 & 0.1319 \\
\hline Pooled deviations. & 377 & 0.4824 & 0.0487 & 0.675 & 0.2390 \\
\hline Pooled error & & & 0.0211 & 0.2758 & 0.1097 \\
\hline$* *$ P $\leq 0.01$ & & & &
\end{tabular}

$* * \mathrm{P} \leq 0.01$. 


\section{Stability measures}

Results on phenotypic stability of the seven genotypes are presented in Table (12). The different stability parameters had inconsistent indications regarding most of the traits. For fresh forage yield MI reya was declared the most stable genotype according to the parameters suggested by Eberhart and Russell (1966). However, the genotype WL903 appeared to be the most stable based on the low variance indicated by the estimates of Wircke (1962) and Shulka (1972), while the genotype Ismailia-1 appeared to be more stable according to the measure of Lin and Binns (1988). The nonparametric method of ranking proposed by Nassar and Huehn (1978) once again favored WL903 as the most stable genotype (Table 12). As to the genotypic stability for the fresh forage yield, Figure (1) plots the relationship between the two estimates $\lambda$ and $\alpha$ according to Tai (1971) and suggests that the genotypes MI reya and WL903 were located in the average stability area, indicating their genetic stability compared to the other genotypes.

In case of dry forage yield, the genotypes MI reya and WL903 were suggested as the most stable genotypes according to Eberhart and Russell (1966). MI reya was declared also to be the most stable genotype according to the measure of Wircke (1962) and as stable as the genotype WL625HQ according to Shulka (1972). The local genotype Ismailia-1 was declared most stable according to Lin and Binns (1988) estimates and to the estimates of Nassar and Huehn (1987), which also considered WL1111 and WL625HQ to be stable genotypes. Based on the results shown in Figure (1) the genotype MI reya, could be considered genetically stable for that trait than the remaining genotypes.

Table 12: Stability parameters of seven alfalfa genotypes across eighteen cuts and their mean values forage green and dry forage yield and protein and fiber percentage.

\begin{tabular}{|c|c|c|c|c|c|c|c|c|}
\hline \multirow{3}{*}{ Genotype } & \multirow{3}{*}{ Mean } & \multicolumn{5}{|c|}{ Parametric stability parameters } & \multicolumn{2}{|c|}{$\begin{array}{c}\text { Non-parametric } \\
\text { stability parameter }\end{array}$} \\
\hline & & \multicolumn{2}{|c|}{ Eberhart \& Russell } & \multirow{2}{*}{$\frac{\text { Wricke }}{\text { Wi }}$} & \multirow{2}{*}{$\frac{\text { Shukla }}{\sigma^{2}}$} & \multirow{2}{*}{$\begin{array}{c}\text { Lin } \\
\mathbf{P i} \\
\end{array}$} & \multicolumn{2}{|c|}{ Nassar \& Huehn } \\
\hline & & bi & $\mathbf{S}^{2} \mathbf{d i}$ & & & & $\mathbf{S i}^{(1)}$ & $\mathbf{S i}^{(2)}$ \\
\hline \multicolumn{9}{|c|}{ Fresh forage yield } \\
\hline Us Stone & $10.167 *$ & $1.348^{*}$ & $0.7117 *$ & 2.7689 & $2.389 * *$ & 3.372 & 0.2 & 4.72 \\
\hline MI reya & $10.078^{*}$ & 0.937 & -0.0369 & 1.0090 & 0.309 & 2.294 & 0.2 & 3.84 \\
\hline WL903 & 9.669 & 1.036 & -0.1821 & 0.6632 & 0.093 & 3.533 & 0.12 & 2.54 \\
\hline WLML9 & 9.747 & $0.887 *$ & 0.3333 & 1.8905 & $0.879 * *$ & 3.586 & 0.31 & 5.07 \\
\hline WL1111 & 9.900 & 1.000 & $1.1394 * *$ & 3.8166 & $1.822 * *$ & 3.555 & 0.22 & 3.01 \\
\hline WL625HO & 9.551 & 1.038 & 0.303 & 1.8206 & $0.734 * *$ & 4.529 & 0.2 & 3.53 \\
\hline Ismailia-1 & $11.089 *$ & $0.753 *$ & $2.1906^{* *}$ & 6.3112 & $3.776^{* *}$ & 1.037 & 0.2 & 4.49 \\
\hline \multicolumn{9}{|c|}{ Dry forage yield } \\
\hline Us Stone & $2.042 *$ & $1.288^{*}$ & 0.054 & 3.9631 & $0.124 * *$ & 0.130 & 0.27 & 5.71 \\
\hline MI reva & $2.036^{*}$ & 0.959 & 0.003 & 1.2736 & 0.020 & 0.089 & 0.21 & 3.29 \\
\hline WL903 & 1.930 & 1.024 & 0.016 & 1.9209 & 0.035 & 0.170 & 0.2 & 3.94 \\
\hline WLML9 & 1.969 & $0.865 *$ & 0.006 & 1.4337 & 0.032 & 0.147 & 0.33 & 4.06 \\
\hline WL1111 & 1.941 & $0.865^{*}$ & 0.051 & 3.7841 & 0.090 & 0.178 & 0.18 & 3.24 \\
\hline WL625HQ & 1.861 & $1.132 *$ & 0.001 & 1.1408 & 0.024 & 0.209 & 0.19 & 2.65 \\
\hline Ismailia-1 & $2.243 *$ & $0.869^{*}$ & $0.066^{*}$ & 4.6060 & 0.110 & 0.041 & 0.2 & 2.53 \\
\hline \multicolumn{9}{|c|}{ Protein percentage } \\
\hline Us Stone & 18.093 & $0.894 *$ & $0.547 *$ & 9.8467 & $0.668 * *$ & 2.830 & 0.18 & 3.29 \\
\hline MI reva & 19.381 & $0.897 *$ & $0.450 *$ & 8.2929 & $0.533 * *$ & 0.682 & 0.19 & 2.65 \\
\hline WL903 & 18.614 & $0.872 *$ & $1.354^{*}$ & 22.7668 & $1.781 * *$ & 2.052 & 0.16 & 4.29 \\
\hline WLML9 & 18.468 & $1.110^{*}$ & $1.174 *$ & 19.8971 & $1.503 * *$ & 2.110 & 0.2 & 3.76 \\
\hline WL1111 & $18.896^{*}$ & 1.066 & 1.369 & 23.0313 & $1.685^{* *}$ & 1.563 & 0.2 & 4.47 \\
\hline WL625HO & 18.586 & 1.063 & $0.883 *$ & 15.2446 & $1.041 * *$ & 2.079 & 0.24 & 3.59 \\
\hline Ismailia-1 & 18.317 & $1.098 *$ & $1.279 * *$ & 21.5741 & $1.616^{* *}$ & 2.725 & 0.24 & 4.53 \\
\hline \multicolumn{9}{|c|}{ Fiber percentage } \\
\hline Us Stone & $22.645 *$ & 1.006 & 0.288 & 11.662 & $0.327 * *$ & 0.475 & 0.24 & 3.65 \\
\hline MI reva & $22.964 *$ & $1.156^{*}$ & $0.459 * *$ & 17.893 & $0.659 * *$ & 0.327 & 0.15 & 3.12 \\
\hline WL903 & 22.526 & 1.003 & $0.644 * *$ & 24.681 & $0.795 * *$ & 0.735 & 0.26 & 4.18 \\
\hline WLML9 & 22.422 & 1.015 & 0.298 & 12.006 & $0.340 * *$ & 0.790 & 0.25 & 3.06 \\
\hline WL1111 & 22.418 & 0.966 & 0.255 & 10.453 & $0.288 * *$ & 0.812 & 0.2 & 3.47 \\
\hline WL625HQ & 22.181 & 0.781 & $0.440^{*}$ & 17.168 & $0.739 * *$ & 1.36 & 0.26 & 5.59 \\
\hline Ismailia-1 & 22.087 & $1.073 *$ & 0.096 & 4.602 & 0.096 & 1.046 & 0.17 & 2.12 \\
\hline
\end{tabular}




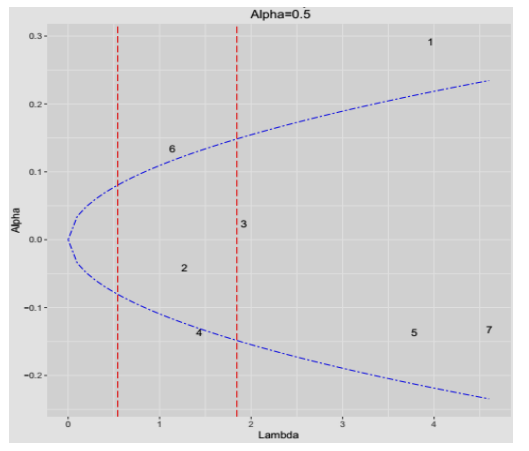

Dry forge yield

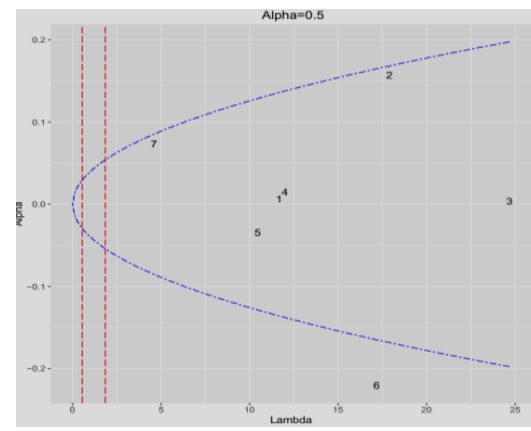

Fiber \%

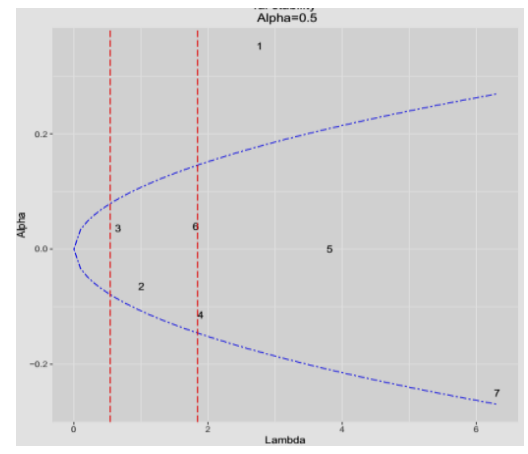

Green forge yield

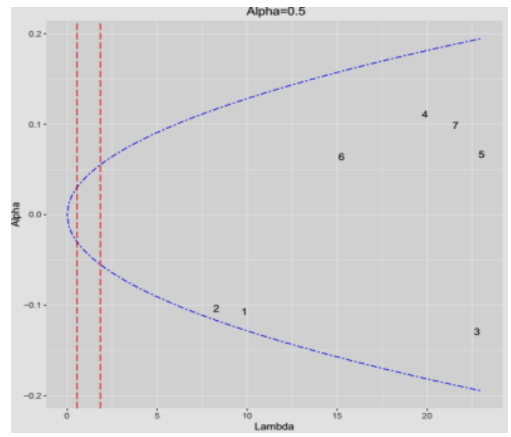

Protein \%

Fig.1: Distribution of estimates of genotypic stability parameters of 7 alfalfa cultivars

Phenotypic stability measures for protein percentage almost showed consensus of the stability of MI reya (Table 12). Similar to Eberhart and Russell (1966) estimates, the genotypic estimates according to Tai (1971), as observed from Figure (1), no obvious stability could be declared among the seven studied genotypes. On the other hand the consensus among the stability parameters were in favor of Ismailia-1 for the fiber percentage except for the estimate of Lin and Binns (1988) that recognized MI reya as the most stable genotype. No genotype could reach the threshold of genetic stability as seen in Figure (1), for the fiber percentage trait.

The results presented here indicated that the local cultivar Ismailia-1 was superior to the introduced genotypes regarding its fresh and dry forage yield in addition to recording the least fiber percentage; however, MI reya produced higher protein percentage. When stability measures for the four traits was considered, Isamilia-1 was considered the most stable for fiber percentage based on four different measures tested. MI reya was most stable for protein percentage and more stable than Ismailia-1 for fresh and dry forage yield based on estimates from larger number of the tested stability measures, suggesting that MI reya is the most promising genotype among the introduced genotypes under study. Ismailia-1 remains the superior genotype due to its significantly higher yields compared to the newly introduced genotypes.

\section{REFERENCES}

Abd El-Galil, M.M. and N.M. Hamed, 2008. Evaluation of yield potential, genetic variances and correlation for nine cultivars of alfalfa under the New Valley environment.J. Agric. Sci., Mansoura Univ. 33: 4771-4776.

Abd El-Halim, A.Z.; I. A. Hanna and T.A. Mahmoud (1992): Productivity and forage quality of some alfalfa cultivars on newly reclaimed sandy soils. Egypt .J. Appl. Sci., 7 (9): 407-427.

Bakheit, B.R. (1988). Variation, correlation and path-coefficient analysis in some world varieties of alfalfa (Medicago sativa L.). Assiut J. of Agric. Sci. 19(5): 149-163.

Allard,R. W. and A. D. Bradshow. 1964. Implication of genetic-environmental interaction in applied plant breeding .Crop Sci. 4: 503-508.

A.O.A.C., 1990. Association of Official Analytical Chemists Methods of Analysis $15^{\text {th }}$ Edition. Washington, D.C. 
Babinec, J., Z. Kozova, , E. Strakova, P. Suchy, 2001. The variance of the amino acids in some Lucerne (Medicago sativa L.) populations. In I. Delgado, \& J. Lloveras, Quality in Lucerne and medics for anomal production (pp. 235-239). Zaragoza (Spain): CIHEAM.

CAPMAS. 2019. Central Agency for Public Mobilization and Statistics. nnual bulletin of statistical crop area and plant production, Egypt. Issue no. 71-22122-2017.

Dale L. M., 2011. Determination of forage quality by destructive and non-destructive methods. $\mathrm{PhD}$ thesis. USAMV Cluj, 330p.

Decruyenaere, V., P. Lecomte, C. Demarquilly, J. Aufrere, P. Dardenne, D. Stilmant, 2008. Evaluation of green forage intake and digestibility in ruminants using near infrared reflectance spectroscopy (NIRS): Developing a global calibration. Animal Feed Science and Technology, 148, 138-156.

Eberhart S.A. and Russell W.A. 1966. Stability parameter for comparing varieties. Crop Sci., 16: 36-40.

Francis, T. R., and L. W. Kannenberg, 1978. Yield stability studies in short-season maize. I. A descriptive method for grouping genotypes. Can. J. Plant Sci., 58(4), 1029-1034.

Finaly, K.W. and G.N. Wilkinson. 1963. The analysis of adaptation in plant- breeding programme. Aust. J. Agric. Res. 14: 742- 754.

Gen stat version $18^{\text {th }}$ edition software (VSN International, 2015).

Heinriches, D., 1970. Variation of chemical constituents within and between alfalfa populations. In M. Norman, $11^{\text {th }}$ Proc. Int. Grassl. Congr. (pp. 267-270). St. Lucia, Queensland: University of Queensland Press.

Lin C.S. and M.R. Binns 1988. A superiority measure of cultivar performance for cultivar $\mathrm{X}$ location data. Canadian J. Plant Sci. 68: 193198.
Nascimento M., L. A.Peternelli, C. D. Cruz, A. C. C. Nascimento, R. P. Ferreira, L. L. Bhering and C. C. Stalgado 2013. Artificial neural networks for adaptability and stability evaluation in alfalfa genotypes. Crop Breeding and Applied biotechnology 13: 152-156, 2013.

Nassar, R. and M. Huehn, 1987. Studies on estimation of phenotypic stability: Tests of significance for non-parametric measures of phenotypic stability. Biometrics 43: 45-53.

Oushy. H.S., O. Niemlainen, M.A. El-Nahrawy and I.A.Hanna (1999). Seasonal variation in performance of alfalfa cultivars under sandy soil condition. 1-Yield and yield components. Egypt. J. plant Breeding. 3: 281-296.

Oushy, H.S., M.M. Abdel-Galil and N.M.Hamd (2007). Performance of local and exotic alfalfa cultivars under different environmental conditions in Egypt. Egypt J. Agric. Res. 85: 2201-2217.

Pinthus, M.J. 1973. Estimates of genotypic value. a proposed method. Euphytica 22: 345-351.

Rammah, A. M., A. E. El-Shahw, M. E. Mousa and I. A. Hanna (1995). Performance of thirteen alfalfa landraces and three local cultivars grown in sandy soil Egypt J. Appl. Sci., 10 (12): 318330.

Stanacev, V., D. Dukic, S. Kovcin, M. Drinic, , N. Puva, V. Stanacev, 2010. Nutritive value of the genetically divergent genotypes of Lucerne (Medicago sativa L.). Africa Journal of Agricultural Research, 5 (11), 1284-187.

SAS. 2014. SAS: Business analytics and business intelligence software. SAS Inst. http://www.sas.com/en_us/home.html (accessed 30 Sept. 2014).

Shukla, G. K. 1972. Genotype stability analysis and its application to potato regional trails. Crop Sci., 11: 184-190.

Snedecor, G. W. and W. G. Cochran. 1989. Statistical Methods. $8^{\text {th }}$ Ed., Iowa State Univ. Press, Ames Iowa, USA.

Tai G.C.C. 1971. Genotypic stability analysis and its application to potato regional trials. Crop Sci., 11: 184-190.

Wricke, G. 1962. Uberiene methode zur erfassung der ökologischen streubreite in eldversuchen. $\mathrm{Z}$. pflanzenzücht, 47: 92-96. 


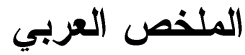

\section{معالم المتوازنة للتفاعل بين التركيب الوراثي والبيئة في بعض التراكيب الوراثية للبرسيم الحجازى}

\author{
مفيدة عبد القادر صيام'، سحر عبد العزيز فرج'

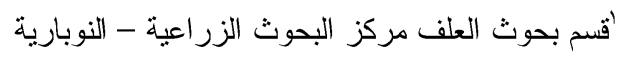 \\ 'المعمل المركزي لبحوث التصميم والنحليل الإحصائي - مركز البحوث الزراعية - الجيزة
}

تهدف هذه الدراسة الى تقييم بعض التراكيب الور اثية المستوردة من البرسيم الحجازى من ناحية الإنتاجية و الجودة مع تقدير بعض معالم الثبات المختلفة. تم تقييم ستة نر اكيب ور اثية بالإضافة الى الصنف المحلى إسماعيلية

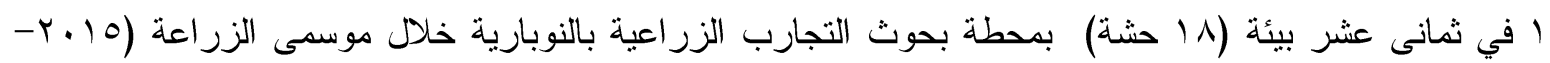

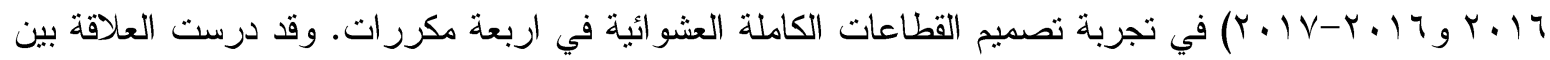
المحصول وأهم عو امله بإستخدام تحليل التباين. إستخدمت خمسة طرق لتقدير معالم الثبات و المظهري وطريقة واحدة لتقدير معالم الثبات الوراثي للصفات تحت الدراسة بهدف إستخدام المعلومات المتاحة من هذه التقديرات لتوجيه بر امج التربية. وكانت الصفات تحت الدراسة هي (محصول العلف الاخضر ومحصول العلف الجاف ونسبة مانة البروتين ونسبة الألياف) ويمكن تلخيص النتائج كما يلى: كان تأثير الصنف و البيئة و التفاعل بينهما عالي المعنوية لجميع الصفات تحت الدراسة ما عدا طول النبات حيث لم يكن التفاعل معنوياً.

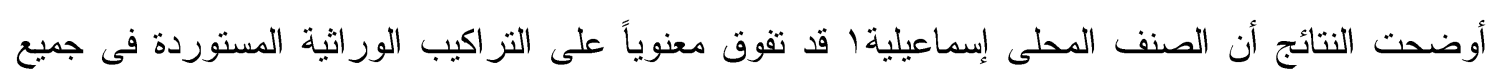

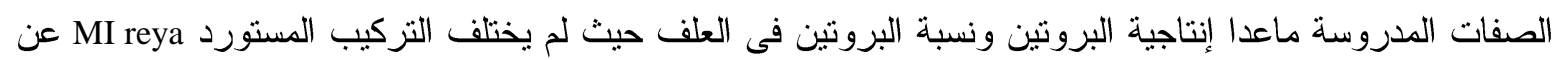
الصنف المحلى معنوياً فى إنتاجية البروتين بل وتفوق MI reya على إسماعيليةا معنوياً فى نسبة البروتين. أما بالنسبة للثبات المظهرى و الوراثى للتر اكيب الور اثية المستوردة والصنف المحلى فقد إتضح وجود تباين كبير بين مقاييس سلوك الثبات المختلفة تحت الدراسة. وبناء على التوافق ما بين أكبر عدد من المقاييس المدروسة يمكن

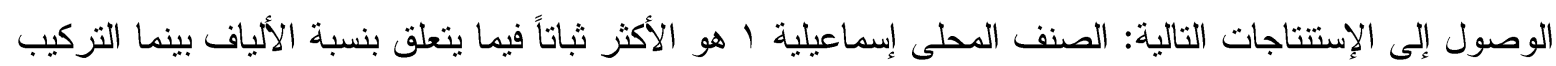
الوراثى MI reya هو الأعلى ثباتاً لصفة نسبة البروتين و يتفوق على الصنف إسماعيلية ا فى الثبات لصفة المحصول الأخضر و الجاف و عليه ينصح بإستعماله في برامج التربية كأصل ور اثي حيث يتمتع بقدرة ثبات مرتفعة. 